\title{
Impact of NHS walk-in centres on primary care access times: ecological study
}

\section{Ravi Maheswaran, senior clinical lecturer in public health medicine, ${ }^{1}$ Tim Pearson, research associate in public health, ${ }^{1}$ James Munro, senior clinical lecturer in epidemiology, ${ }^{2}$ Moyez Jiwa, clinical lecturer in general practice research, ${ }^{3}$ Michael J Campbell, professor of medical statistics, ${ }^{2}$ Jon Nicholl, professor of health services research ${ }^{2}$}

${ }^{1}$ Public Health GIS Unit, School of Health and Related Research, University of Sheffield, Sheffield S1 4DA

${ }^{2}$ Health Services Research Unit, School of Health and Related Research, University of Sheffield ${ }^{3}$ Institute of General Practice, School of Health and Related Research, University of Sheffield

Correspondence to: R Maheswaran r.maheswaran@sheffield.ac.uk

doi: 10.1136/bmj.39122.704051.55 on access to primary care by freeing up time during

\section{ABSTRACT}

Objective To examine whether walk-in centres contribute to shorter waiting times for a general practice

appointment.

Design Ecological study.

Setting 2509 general practices in 56 primary care trusts in England; 32 walk-in centres within $3 \mathrm{~km}$ of one of these practices.

Main outcome measure Waiting time to next available general practitioner appointment (April 2003 to

December 2004), from national monthly primary care access survey.

Results The percentage of practices achieving the target waiting time of less than 48 hours to see a general practitioner increased from $67 \%$ to $87 \%$ over the 21 month study period (adjusted odds ratio 1.07 (95\% confidence interval 1.06 to 1.08) per increase in month). Achievement of the waiting time target decreased with increasing multiple deprivation (0.57 (0.49 to 0.67) for most versus least deprived third) and increased with increasing practice population size (1.02 (1.00 to 1.04 ) per 1000 increase). No evidence was found that increasing distance from a walk-in centre was associated with decreasing odds of achieving the waiting time target (1.00 (0.99 to 1.01) per km increase). Increasing "exposure" to a walk-in centre, modelled with a distance decay function based on attendance rates, also showed little evidence of association with achievement of the waiting time target (1.02 (0.97 to 1.08) for interquartile range increase). No evidence existed that the rate of increase in achieving the 48 hour target over time was enhanced by proximity or "exposure" to a walk-in centre. Results were similar when the analysis was rerun with data for 2003 only (done because pressure in 2004 to meet the government's deadline might have led to other changes that could have masked any walk-in centre effect).

Conclusions No evidence existed that walk-in centres shortened waiting times for access to primary care, and the results do not support the use of walk-in centres for this purpose.

\section{INTRODUCTION}

Forty two National Health Service walk-in centres opened in England in 2000-3, and a further 21 opened in 2004. They are primarily nurse led, have wide opening hours, and provide information and treatment for minor conditions without the need for appointments. One of their aims is to relieve the pressure normal general practice surgery hours for patients who need to see their general practitioner. ${ }^{1}$ The government has seen waiting times for treatment, including delays in access to primary care, as the public's principal concern about the NHS. ${ }^{2}$

The introduction of walk-in centres as an additional point of first contact with the NHS received a mixed reception; some people were concerned that it would increase demand rather than reduce the workload for primary care. ${ }^{3}$ The evidence to date is inconclusive; one study of 10 walk-in centres reported non-significant reductions in consultation rates with general practitioners in practices within $3 \mathrm{~km}$ of these centres, ${ }^{4}$ whereas another study of a single walk-in centre found no evidence of an impact on consultation rates or waiting times for an appointment with a general practitioner. $^{5}$

To reduce waiting times for access to primary care, the government introduced a target waiting time and expected patients waiting for an appointment with a general practitioner to be seen within 48 hours, with a deadline of 2004 for achieving this target. ${ }^{2}$ A national primary care access survey carried out once monthly from April 2003 monitored progress towards the target. Our aim was to examine whether walk-in centres contributed to shorter waiting times to see a general practitioner in practices situated close to walk-in centres.

\section{METHODS}

We defined the geographical areas for the study as 76 primary care trusts in England. We set out to include all general practices within these primary care trusts and examine the effect of all walk-in centres within and surrounding these primary care trusts. The ecological study was part of a wider project that included a qualitative phase, the results of which will be reported separately. (For the qualitative phase, we chose the first four walk-in centres to open in 2004 and four established walk-in centres in the same regions of the country 
with similar characteristics in terms of their focus on primary care. The primary care trusts for the ecological study were all those within a $20 \mathrm{~km}$ radius of the eight walk-in centres; we must emphasise that this ecological analysis examined the effect of all walk-in centres in and surrounding the study areas and was not confined to the eight walk-in centres included in the qualitative study.)

The outcome measure was waiting time in days to the next available appointment with a general practitioner. This was the value reported in the national primary care access survey, in which primary care trusts telephoned all practices in their area on a predetermined weekday once a month to record the date of the next available surgery appointment with a general practitioner. ${ }^{6}$ For example, if the survey was carried out on a Wednesday and the next available appointment was the following Monday, the wait would be recorded as three days. A validation survey found no overall reporting bias towards shorter waiting times. ${ }^{7}$ We used survey data from April 2003 to December 2004.

We calculated two measures of "exposure" of practices to walk-in centres. The first was the distance in a straight line from a practice to the nearest walk-in centre that had opened before the survey was carried out for that month. The size of the effect of walk-in centres on a practice's waiting time will depend on how many patients from the practice attend the walk-in centre. However, although attendance data for walk-in centres included a field for the patients' general practitioner, this was largely left blank and we could not calculate the proportion of patients attending from any particular general practice. Instead, we used a function based on walk-in centre attendance rates by distance as a second measure of exposure to walk-in centres.

We used attendance data from 2003-4, which included output area of residence from the 2001 census, for the four established walk-in centres to calculate attendance rates by using $1 \mathrm{~km}$ concentric rings around the walk-in centres, with output area population counts from the 2001 census as the denominator. (No electronic data were available for the four new walk-in centres.) A total of 167045 people (first attendance in the two year period) attended the four walk-in centres, and $78-91 \%$ lived within $6 \mathrm{~km}$ of the walk-in centre they attended. The attendance rates by distance show a marked fall in rates with increasing distance from a walk-in centre (fig 1). We fitted an exponential distance decay function to the attendance rates $\left(y=428.78 \mathrm{e}^{-0.56 \mathrm{x}}\right.$, where $x$ represents the straight line distance $(\mathrm{km})$ to the walk-in centre). Thus, for people living $1 \mathrm{~km}$ from a walk-in centre the predicted attendance rate would be $428.78 \mathrm{e}^{-0.56^{*} 1}=245$ per 1000 population over two years. We used the function to calculate a spatially summed distance decay value for each general practice by month on the basis of its distance to each open walkin centre. General practices near to more than one walk-in centre may have a greater potential exposure, and we calculated the spatially summed value by month to take into account the potential influence from multiple walk-in centres in the vicinity of a general practice.

We attached an index of multiple deprivation 2004 score - a widely used, nationally available area level deprivation score - to each practice on the basis of the lower layer super output area in which the practice was located. ${ }^{8}$ The lower layer super output area is the smallest geographical area for which this deprivation index is routinely available and typically comprises four to six 2001 census output areas. As $97.6 \%$ of practices were situated in urban areas, we did not examine urban-rural status in any analyses. We obtained the number of registered patients for each practice from primary care trusts. (List size breakdown by age and sex and number of whole time equivalent general practitioners were too incomplete for use in the analyses).

\section{Statistical analysis}

We analysed the data with waiting time as a binary outcome ( $<2$ days $v \geq 2$ days) to reflect the 48 hour government target. The logistic regression models incorporated deprivation categorised by national super output area thirds and practice list size and time (month) as continuous variables. We examined distance and the distance decay value as continuous variables and as categorical variables (distance: $<3,3$ to $<6$, $\geq 6 \mathrm{~km}$; distance decay value grouped by thirds) in separate models. We included general practice as a random effect to adjust for clustering of waiting times by practice. We used generalised estimating equations (Genmod procedure) in SAS (release 9.1; S AS Institute, Cary, NC, 2002) to do the analyses.

We then examined if exposure to walk-in centres influenced the rate at which the waiting time target was achieved over the study period by incorporating interaction terms for month by walk-in centre exposure. To examine if a delay between walk-in centres opening and becoming operationally effective had any influence on the results, we reran analyses after adding a 30 day lag to walk-in centres' opening dates. As pressure to meet the government's deadline of December 2004 might have led to other changes that could have masked any effect of walk-in centres, we also reran the analysis using data for 2003 only.

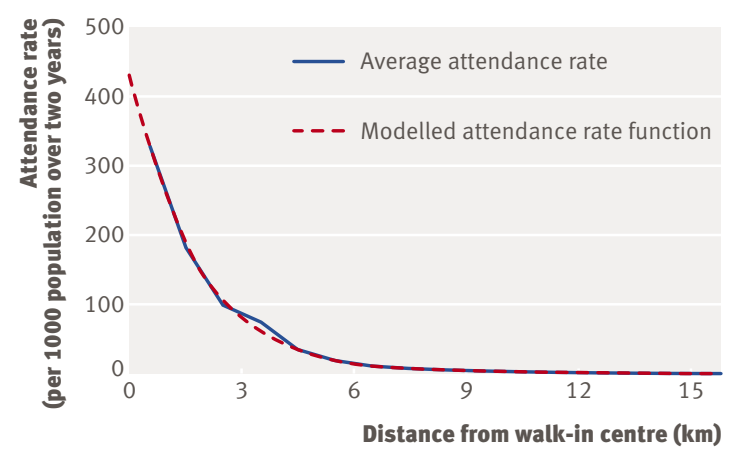

$\overline{\text { Fig } 1 \text { | Walk-in centre attendance rates by distance from walk-in }}$ centre based on data on people attending four established walk-in centres in England, 2003-4 


\section{RESULTS}

We received information from 56 of 76 primary care trusts (74\% response) and were supplied with primary care access survey data for 2509 of the 2564 practices within these 56 primary care trusts. (No access times were recorded for practices that did not operate an appointment system.) Thirty two of the 63 walk-in centres that were in operation by December 2004 were within $3 \mathrm{~km}$ of at least one of these 2509 practices; $483(19 \%)$ of the 2509 practices were within $3 \mathrm{~km}$ of a walk-in centre open in April 2003, and this increased to $879(35 \%)$ by December 2004. Waiting times were missing for $4.5 \%$ of practice months over the 21 month study period. List size was missing for 174 $(6.9 \%)$ practices. Median list size was 4476 (interquartile range 2850-7200).

A clear decrease in waiting times to see a general practitioner occurred over the 21 months (fig 2). In April 2003, 67\% of practices had a waiting time of less than 48 hours; this increased to $87 \%$ by December 2004. Table 1 shows the unadjusted percentages of practices that achieved the waiting time target in April 2003 and December 2004 by deprivation, list size, distance from a walk-in centre, and "exposure" in terms of the distance decay value; table 2 shows the adjusted odds ratios for achieving the target waiting time. (The odds ratios should not be interpreted as an

\begin{abstract}
Table 1 | Percentage of practices that achieved the waiting time target of less than 48 hours by multiple deprivation, practice list size, distance from the nearest walk-in centre, and distance decay value. Values are percentages (numbers) unless stated otherwise
\end{abstract}

\begin{tabular}{|c|c|c|c|}
\hline \multirow[b]{2}{*}{ Variables and categories } & \multirow{2}{*}{$\begin{array}{l}\text { No of practices } \\
(n=2509)\end{array}$} & \multicolumn{2}{|c|}{ Practices achieving $₫ 48$ hour target ${ }^{\star}$} \\
\hline & & April 2003 & December 2004 \\
\hline \multicolumn{4}{|c|}{ Index of multiple deprivation (by national super output area thirds) } \\
\hline Most deprived & 1395 & $63(788 / 1247)$ & $86(1135 / 1313)$ \\
\hline Intermediate & 666 & $68(430 / 635)$ & $86(531 / 620)$ \\
\hline Least deprived & 448 & $75(321 / 426)$ & $92(401 / 436)$ \\
\hline \multicolumn{4}{|l|}{ List size (by thirds) $\dagger$} \\
\hline$\geq 6050$ & 778 & $66(490 / 742)$ & $90(673 / 751)$ \\
\hline $3301-16050$ & 779 & $65(473 / 724)$ & $88(662 / 756)$ \\
\hline$\ll 3301$ & 778 & $68(471 / 692)$ & $85(618 / 730)$ \\
\hline \multicolumn{4}{|c|}{ Distance from nearest walk-in centre } \\
\hline$\ll 3 \mathrm{~km}$ & 483 & $63(277 / 440)$ & - \\
\hline $3-46 \mathrm{~km}$ & 734 & $64(414 / 650)$ & - \\
\hline$\geq 6 \mathrm{~km}$ & 1292 & $70(848 / 1218)$ & - \\
\hline $33 \mathrm{~km}$ & 879 & - & $85(709 / 830)$ \\
\hline $3-46 \mathrm{~km}$ & 792 & - & $89(672 / 756)$ \\
\hline$\geq 6 \mathrm{~km}$ & 838 & - & $88(686 / 783)$ \\
\hline \multicolumn{4}{|c|}{ Distance decay value $\ddagger$ (by thirds based on all months) } \\
\hline$\geq 76.1$ & 660 & $62(367 / 591)$ & - \\
\hline $9.3-<76.1$ & 834 & $66(505 / 760)$ & - \\
\hline$\langle 9.3$ & 1015 & $70(667 / 957)$ & - \\
\hline$\geq 76.1$ & 1205 & - & $86(981 / 1140)$ \\
\hline $9.3-<76.1$ & 749 & - & $87(622 / 716)$ \\
\hline <9.3 & 555 & - & $90(464 / 513)$ \\
\hline
\end{tabular}

*Denominators are lower than number of practices owing to missing data on waiting times.

†List size missing for 174 practices.

$\ddagger$ Summed value from all walk-in centres based on distance from walk-in centres and modelled attendance rate (see methods section for details); high value=high "exposure."



$\overline{\text { Fig } 2 \text { | Relative frequency distribution of waiting time (days) to }}$ the next available general practitioner appointment at 2509 general practices in the study areas in England, April 2003 to December 2004

approximation to relative risk because of the high proportions that achieved the waiting time target.)

The odds of achieving a waiting time of less than 48 hours decreased with increasing deprivation. A practice in the most deprived category had an adjusted odds ratio of 0.57 (95\% confidence interval 0.49 to $0.67)$ relative to a practice in the least deprived category. The odds of a waiting time of less than 48 hours increased over the period of study, with an adjusted odds ratio of 1.07 (1.06 to 1.08) per month. A larger registered practice population was associated with higher odds of achieving a waiting time of less than 48 hours, with an adjusted odds ratio of 1.02 (1.00 to $1.04)$ per 1000 increase in list size.

We found no evidence that increasing distance from a walk-in centre was associated with decreasing odds of achieving the 48 hour waiting time target (adjusted odds ratio 1.00 (0.99 to 1.01) per km increase). Similarly, no evidence existed to suggest that increasing exposure to a walk-in centre in terms of the distance decay value was associated with increasing odds of achieving a waiting time under 48 hours (odds ratio 1.02 (0.97 to 1.08) for an interquartile range increase). We also found no evidence of association when we examined distance and distance decay as categorical variables.

No evidence existed that the rate of increase in achieving the 48 hour target over time was enhanced by exposure to a walk-in centre. Results were similar with lagged walk-in centre opening dates and with the analysis restricted to 2003 (data not shown).

\section{DISCUSSION}

A clear increase occurred in the percentage of practices that achieved the target waiting time of less than 48 hours to see a general practitioner over the 21 month study period, but we found no evidence that walk-in centres contributed to shorter waiting times for access to primary care. Waiting times were longer in more socioeconomically deprived areas and shorter in larger practices. 


\section{Limitations}

One possible explanation for the apparent lack of effect of walk-in centres is that an effect existed but was not detected by our study. This could have been for several reasons. The once monthly survey may be an insensitive measure of waiting time. If workload increased in a practice, partners might have added extra surgeries and waiting times may have changed very little. As practices were monitored on their performance at reducing waiting times, we cannot rule out the possibility that extra appointments were opened up around dates of the waiting time survey to reduce waiting times. However, the validation survey carried out previously found no overall reporting bias towards shorter waiting times. ${ }^{7}$ In addition, when we restricted the analysis to 2003, when there would have been less pressure to meet the December 2004 target, no walk-in centre effect was evident.

A further limitation relates to the assessment of exposure of practices to walk-in centres. As information on walk-in centre patients' general practitioners was largely missing, we could not use this information and had to rely instead on proximity measures. People attending some walk-in centres, particularly in commuter cities, may live a long way away and any effect on waiting times for their general practitioner would be difficult to detect. However, the walk-in centre attendance data, at least for the four walk-in centres examined, showed that a high proportion of attenders lived close to the walk-in centres. The incomplete information on general practitioners also meant that we could

Table 2 Effects of multiple deprivation, time, general practice list size, and exposure to walk-in centres on odds of achieving 48 hour primary care access waiting time target in 2509 practices in the study areas in England, April 2003 to December 2004

\begin{tabular}{|c|c|c|}
\hline Variable & Adjusted odds ratio* $(95 \% \mathrm{Cl})$ & Significance \\
\hline \multicolumn{3}{|l|}{ Deprivation (by third): } \\
\hline Most deprived & $0.57(0.49$ to 0.67$)$ & \multirow[t]{3}{*}{$x^{2}=54.7, d f=2 ; P<0.0001$} \\
\hline Intermediate & $0.71(0.59$ to 0.84$)$ & \\
\hline Least deprived & 1 & \\
\hline Time (months) & 1.07 (1.06 to 1.08$)$ & $x^{2}=468.5, d f=1 ; P<0.0001$ \\
\hline List size (in 1000s) & $1.02(1.00$ to 1.04$)$ & $X^{2}=6.6, d f=1 ; P=0.01$ \\
\hline $\begin{array}{l}\text { Distance from nearest walk- } \\
\text { in centre (per km increase) }\end{array}$ & $1.00(0.99$ to 1.01$)$ & $X^{2}=0.02, d f=1 ; P=0.90$ \\
\hline \multicolumn{3}{|c|}{ Distance from nearest walk-in centre (by category): } \\
\hline$\ll 3 \mathrm{~km}$ & $1.01(0.89$ to 1.16$)$ & \multirow[t]{3}{*}{$X^{2}=5.24, d f=2 ; P=0.07$} \\
\hline $3-\ll 6 \mathrm{~km}$ & $0.89(0.79$ to 1.00$)$ & \\
\hline$\geq 6 \mathrm{~km}$ & 1 & \\
\hline $\begin{array}{l}\text { Distance decay value } \neq \\
\text { (interquartile range increase) }\end{array}$ & $1.02(0.97$ to 1.08$)$ & $X^{2}=0.5, d f=1 ; P=0.47$ \\
\hline \multicolumn{3}{|l|}{ Distance decay value (by thirds): } \\
\hline High exposure & $1.00(0.88$ to 1.14$)$ & \multirow[t]{3}{*}{$X^{2}=0.02, d f=2 ; P=0.99$} \\
\hline Intermediate & $1.00(0.89$ to 1.12$)$ & \\
\hline Low exposure & 1 & \\
\hline
\end{tabular}

Waiting time was modelled as the odds of having a waiting time of $<48$ hours ( $v \geq 48 \mathrm{~h}$ ).

*From logistic regression models that included deprivation, time, list size, and a measure of exposure to walk-in centres (either distance or distance decay as continuous or categorical variable in separate models), with

general practice as a random effect; odds ratios should not be interpreted as an approximation to relative risk

because proportions achieving the waiting time target are high. Relative risk may be estimated with the formula:

$R R=O R /\left[(1-P 0)+\left(P_{0} * O R\right)\right]$ where $R R=$ relative risk, $O R=$ odds ratio, and $\mathrm{Po}=$ proportion in reference category.

$\ddagger$ See methods section for details. not assess the extent to which patients attending walkin centres were not registered with a general practitioner. The study was able to detect improvements in waiting time over the 21 month study period, longer waiting times in deprived areas, and shorter waiting times in practices with larger list sizes, although these effects are likely to be much stronger than any walk-in centre effect.

\section{Interpretation of results}

Another interpretation of the results is that walk-in centres had little impact on waiting times for access to primary care. Several possible explanations exist for this apparent lack of effect. Walk-in centres may have mainly extended the role of first contact services rather than offering an alternative to general practitioners for some conditions; they may have created more demand and seen patients who would otherwise not have attended for health care. Evidence exists that the age, sex, ethnic, and socioeconomic profile of walk-in centre attenders is slightly different from that of general practitioner attenders. ${ }^{9}$ Duplication of services could have arisen for several reasons. Patients may have used walk-in centres as a source of a second opinion or to gain reassurance. They may have been referred inappropriately to walk-in centres and have had to go back to see their general practitioners. In addition, some nurses in walk-in centres may not have had the skill levels needed and may have sent patients back to the general practitioner.

Our results are consistent with the results of Hsu and colleagues, who found no effect of a single walk-in centre on workload or waiting times in nearby practices. In addition, in a survey of users of walk-in centres, $13 \%$ were referred back to their practice and a further $32 \%$ intended to make an appointment with their general practitioner. ${ }^{9}$ In a survey of healthcare providers, one third of respondents felt that patients' expectations had increased and 15\% felt that workload had also increased since their local walk-in centre opened. ${ }^{10}$ An interview study with users of walk-in centres found that walk-in centres functioned as a way for people to use the NHS without feeling that they were increasing the burden on what they perceived to be overstretched general practice facilities. ${ }^{11}$ Interviews with managers and nurses found no consensus on the core competencies needed by nurses working in first wave walk-in centres and as a result no standardisation of induction, training, or support for walk-in centre nurses. ${ }^{12}$

\section{Conclusion}

Walk-in centres are part of an increasingly complex network of primary care and first contact services for health care and may extend and at times potentially duplicate rather than offer an alternative to care provided by general practitioners. We found no evidence that walk-in centres shortened waiting times for access to primary care, and our study does not support the use of walk-in centres for this purpose. 


\section{WHAT IS ALREADY KNOWN ON THIS TOPIC}

NHS walk-in centres are primarily nurse led, have wide opening hours, and provide information and treatment for minor conditions without the need for appointments

One of their aims is to relieve the pressure on access to primary care by freeing up time during normal surgery hours for patients who need to see their general practitioner

Concerns exist that they increase demand rather than reduce the workload for primary care, but the evidence to date is inconclusive

\section{WHAT THIS STUDY ADDS}

No evidence existed that walk-in centres shortened waiting times for access to primary care, and the results do not support the use of walk-in centres for this purpose

Contributors: JM, JN, RM, and MJ had the idea for the study. TP and RM carried out the study. MJC advised on statistical analysis. RM drafted the paper with contributions from all authors and is the guarantor.

Funding: Department of Health (Policy Research). The views expressed are those of the authors and not necessarily those of the Department of Health. Competing interests: None declared.

Ethical approval: Metropolitan Multi-centre Research Ethics Committee (04) MRE11/4).

1 Department of Health. Up to $£ 30$ million to develop 20 fast access walkin centres. London: Department of Health, 1999. (Press release 1999/0226.)

2 Department of Health. Delivering the NHS plan: next steps on investment, next steps on reform. London: Department of Health, 2002.
3 Royal College of General Practitioners. Discussion paper on the implications for general practice of NHS Direct and walkin centres. London: RCGP/NHS Alliance, 1999.

4 Chalder M, Sharp D, Moore L, Salisbury C. Impact of NHS walk-in centres on the workload of other local healthcare providers: time series analysis. BMJ 2003;326:532-4.

5 Hsu RT, Lambert PC, Dixon-Woods M, Kurinczuk JJ. Effect of NHS walkin centre on local primary healthcare services: before and after observational study. BMJ 2003;326:530-2.

6 Department of Health. Primary care 24/48 access survey (ROCR/OR/ 0120). Available at: www.dh.gov.uk.

7 Department of Health. DH/PMDU Joint Working_PCAS validation. www.dh.gov.uk/assetRoot/04/07/32/57/04073257.pdf.

8 Office of Deputy Prime Minister. Super output area (lower layer) indices of deprivation 2004 (revised). www.communities.gov.uk/ indices.

9 Salisbury C, Manku-Cott T, Moore L, Chalder M, Sharp D. Questionnaire survey of users of NHS walk-in centres: observational study. Br J Gen Pract 2002;52:554-60

10 Pope C, Chalder M, Moore L, Salisbury C. What do other local providers think of NHS walk-in centres? Results of a postal survey. Public Health 2005;119:39-44.

11 Jackson CJ, Dixon-Woods M, Hsu R, Kurinczuk JJ. A qualitative study of choosing and using an NHS walk-in centre. Fam Pract 2005;22:269-74.

12 Rosen R, Mountford L. Developing and supporting extended nursing roles: the challenges of NHS walk-in centres. J Adv Nurs 2002;39:241-8

Accepted: 24 January 2007 